\title{
CONTINUUM MODEL OF THE TWO-COMPONENT BECKER-DÖRING EQUATIONS
}

\author{
ALI REZA SOHEILI
}

Received 3 April 2003

\begin{abstract}
The process of collision between particles is a subject of interest in many fields of physics, astronomy, polymer physics, atmospheric physics, and colloid chemistry. If two types of particles are allowed to participate in the cluster coalescence, then the time evolution of the cluster distribution has been described by an infinite system of ordinary differential equations. In this paper, we describe the model with a second-order two-dimensional partial differential equation, as a continuum model.
\end{abstract}

2000 Mathematics Subject Classification: 65M20, 82C80, 70F45.

1. Introduction. The Becker-Döring equations model the behavior of clusters of particles when the coagulation and fragmentation done by only one particle may leave or join a cluster at a time [2]. The time evolution of the cluster distribution $c_{r}(t)$ has been described by the infinite system of ordinary differential equations (ODEs) [1]

$$
\dot{c}_{r}=\frac{1}{2} \sum_{k=1}^{r-1} J_{r-k, k}-\sum_{k=1}^{\infty} J_{r, k}, \quad r=1,2, \ldots
$$

where $J_{r, k}=a_{r, k} c_{r} c_{k}-b_{r, k} c_{r+k}=J_{k, r}$, and for $r=1$, the first sum is omitted. $J_{r, k}$ is the net rate of converting the clusters with $r$-particles ( $r$-clusters) to $(r+k)$-clusters, with nonnegative symmetric constants $a_{r, k}$ and $b_{r, k}$ which determine the coagulation and fragmentation rates, respectively. If two different types of particles participated in the cluster coalescence, then the two-component Becker-Döring system is defined based on the following hypotheses:

(1) the number of particles of each type over all clusters is constant;

(2) the clusters are distributed uniformly in space;

(3) the cluster size distribution changes when clusters coagulate or fragment by gaining or losing only monomers of each type.

2. Two-component Becker-Döring system. The concentration of clusters containing $r$ type-I particles and $s$ type-II particles at time $t$ is denoted by $c_{r, s}(t)$. There are two net rates for converting the clusters. Hence, $c_{1,0}(t)$ and $c_{0,1}(t)$ are the concentrations of type-I and type-II monomers, respectively. $J_{r, s}$ is the rate at which $(r, s)$-clusters change to $(r+1, s)$-clusters, and $J_{r, s}^{\prime}$ is the rate at which $(r, s)$-clusters alter to $(r, s+1)$-clusters. 
They are defined by

$$
\begin{aligned}
& J_{r, s}=a_{r, s} c_{r, s} c_{1,0}-b_{r+1, s} c_{r+1, s}, \\
& J_{r, s}^{\prime}=a_{r, s}^{\prime} c_{r, s} c_{0,1}-b_{r, s+1}^{\prime} c_{r, s+1},
\end{aligned}
$$

where $a_{r, s}, b_{r, s}$ are the kinetic coefficients (coagulation and fragmentation rates) for the particle of type I and $a_{r, s}^{\prime}, b_{r, s}^{\prime}$ are the coagulation and fragmentation rates of particles of type II. All coefficients are nonnegative constants with $b_{1,0}=0, b_{0,1}^{\prime}=0$. The equations can be formulated as follows:

$$
\begin{aligned}
& \dot{c}_{r, s}=J_{r-1, s}+J_{r, s-1}^{\prime}-J_{r, s}-J_{r, s}^{\prime}, \quad r \geq 1, s \geq 1, \\
& \dot{c}_{r, 0}=J_{r-1,0}-J_{r, 0}-J_{r, 0}^{\prime}, \quad r \geq 2, \\
& \dot{c}_{0, s}=J_{0, s-1}^{\prime}-J_{0, s}-J_{0, s}^{\prime}, \quad s \geq 2, \\
& \dot{c}_{1,0}=-J_{1,0}-J_{1,0}^{\prime}-\sum_{(r, s) \in I_{f}} J_{r, s}, \\
& \dot{c}_{0,1}=-J_{0,1}-J_{0,1}^{\prime}-\sum_{(r, s) \in I_{f}} J_{r, s}^{\prime},
\end{aligned}
$$

where

$$
I_{f}=\{(r, s), r=0,1,2, \ldots, s=0,1,2, \ldots\}-\{(0,0)\}
$$

The densities of the system are defined by

$$
\rho_{\mathrm{I}}=\sum_{(r, s) \in I_{f}} r c_{r, s}, \quad \rho_{\mathrm{II}}=\sum_{(r, s) \in I_{f}} s c_{r, s}
$$

The two-component Becker-Döring system is formulated as an infinite system of ODEs and so for numerical approximation, the system has to be truncated $[4,5]$.

For the truncated model, we set $J_{r, s}=J_{r, s}^{\prime}=0$ for $r \geq n_{r}$ and $s \geq n_{s}$, where $n_{r}$ and $n_{s}$ are the maximum numbers of particles of type I and type II that can be collected in the clusters. We can use the truncated formulation of the system and obtain

$$
\sum_{s=0}^{n_{s}} \sum_{r=0}^{n_{r}} r \dot{c}_{r, s}(t)=J_{0,1}+2 J_{1,0}+J_{1,0}^{\prime}+\sum_{s=0}^{n_{s}-1} \sum_{r=0}^{n_{r}-1} J_{r, s}=-\dot{c}_{1,0}(t), \quad r+s \geq 2
$$

Hence, $\sum_{s} \sum_{r} r c_{r, s}(t)$ has a constant value of time $t$. The same holds for $\sum_{s} \sum_{r} s c_{r, s}(t)$. On the other hand, the first summation is the number of type-I particles in the overall clusters which we call density of type-I of particles in the system and is denoted by $\rho_{\mathrm{I}}$; so

$$
\rho_{\mathrm{I}}=\sum_{r, s} r c_{r, s}, \quad \rho_{\mathrm{II}}=\sum_{r, s} s c_{r, s}
$$


3. PDE formulation. For both types of particles, the fragmentation coefficients are

$$
b_{r, s}=\frac{a_{r-1, s} Q_{r-1, s}}{Q_{r, s}}, \quad b_{r, s}^{\prime}=\frac{a_{r, s-1}^{\prime} Q_{r, s-1}}{Q_{r, s}} .
$$

In this paper, we have the following kinetic coefficients [4]:

$$
\begin{aligned}
a_{r, s} & =a_{r, s}^{\prime}=1, \\
Q_{r, s} & =\exp \left[-a(r-1)^{2 / 3}-b(s-1)^{2 / 3}\right] .
\end{aligned}
$$

Now using the definitions of $J_{r, s}$ and $Q_{r, s}$, we have

$$
\frac{J_{r, s}}{a_{r, s} Q_{r, s} c_{1,0}^{r+1}}=\frac{c_{r, s}}{Q_{r, s} c_{1,0}^{r}}-\frac{c_{r+1, s}}{Q_{r+1, s} c_{1,0}^{r+1}},
$$

and similarly,

$$
\frac{J_{r, s}^{\prime}}{a_{r, s}^{\prime} Q_{r, s} c_{0,1}^{s+1}}=\frac{c_{r, s}}{Q_{r, s} c_{0,1}^{s}}-\frac{c_{r, s+1}}{Q_{r, s+1} c_{0,1}^{s+1}} .
$$

Thus, we can approximate $J_{r, s}, J_{r, s}^{\prime}$ as follows:

$$
\begin{aligned}
& J_{r, s} \approx-a_{r, s} Q_{r, s} c_{1,0}^{r+1} \frac{\partial}{\partial r}\left(\frac{c_{r, s}}{Q_{r, s} c_{1,0}^{r}}\right), \\
& J_{r, s}^{\prime} \approx-a_{r, s}^{\prime} Q_{r, s} c_{0,1}^{s+1} \frac{\partial}{\partial s}\left(\frac{c_{r, s}}{Q_{r, s} c_{0,1}^{s}}\right) .
\end{aligned}
$$

In this case, all the parameters of the system are functions of nonnegative continuous variables $r, s$. On the other hand, we have

$$
\begin{aligned}
& J(r, s)=-a(r, s) Q(r, s) c(1,0)^{r+1} \frac{\partial}{\partial r}\left(\frac{c(r, s)}{Q(r, s) c(1,0)^{r}}\right), \\
& J^{\prime}(r, s)=-a^{\prime}(r, s) Q(r, s) c(0,1)^{s+1} \frac{\partial}{\partial s}\left(\frac{c(r, s)}{Q(r, s) c(0,1)^{s}}\right) .
\end{aligned}
$$

The system formulation can be stated as follows (assuming $\Delta r=\Delta s=1$ ):

$$
\begin{aligned}
& \dot{c}(r, s)=-\frac{\partial}{\partial r} J(r, s)-\frac{\partial}{\partial s} J^{\prime}(r, s), \quad r \geq 1, s \geq 1, \\
& \dot{c}(r, 0)=-\frac{\partial}{\partial r} J(r, 0)-J^{\prime}(r, 0), \quad r>1, \\
& \dot{c}(0, s)=-J(0, s)-\frac{\partial}{\partial s} J^{\prime}(0, s), \quad s>1 .
\end{aligned}
$$

3.1. Truncated model. Assume $J_{r, s}=J_{r, s}^{\prime}=0$ for $r>n_{r}$ or $s>n_{s}$, where $n_{r}, n_{s}$ are the largest numbers of particles of type I and II which can be collected in one cluster. The truncated model is restricted on the area $\mathbf{R}=\left\{(r, s), 0<r<n_{r}, 0<s<n_{s}\right\}$ and can be formulated as follows:

$$
\frac{\partial c}{\partial t}(r, s, t)=-\frac{\partial J}{\partial r}(r, s, t)-\frac{\partial J^{\prime}}{\partial s}(r, s, t) \quad(r, s) \in \mathbf{R}, t>0 .
$$


The boundary conditions are

$$
\begin{gathered}
\frac{\partial c}{\partial t}(0, s, t)=-J(0, s, t)-\frac{\partial J^{\prime}}{\partial s}(0, s, t), \quad 0<s<n_{s}, \\
\frac{\partial c}{\partial t}\left(r, n_{s}, t\right)=-\frac{\partial J}{\partial r}\left(r, n_{s}, t\right)-J^{\prime}\left(r, n_{s}^{-}, t\right), \quad 0<r<n_{r}, \\
\frac{\partial c}{\partial t}\left(n_{r}, s, t\right)=-J\left(n_{r}^{-}, s, t\right)-\frac{\partial J^{\prime}}{\partial s}\left(n_{r}, s, t\right), \quad 0<s<n_{s}, \\
\frac{\partial c}{\partial t}(r, 0, t)=-\frac{\partial J}{\partial r}(r, 0, t)-J^{\prime}(r, 0, t), \quad 0<r<n_{r},
\end{gathered}
$$

and for the area vertices,

$$
\begin{aligned}
\frac{\partial c}{\partial t}\left(n_{r}, 0, t\right) & =J\left(n_{r}^{-}, 0, t\right)-J^{\prime}\left(n_{r}, 0, t\right), \\
\frac{\partial c}{\partial t}\left(0, n_{s}, t\right) & =J^{\prime}\left(0, n_{s}^{-}, t\right)-J\left(0, n_{s}, t\right), \\
\frac{\partial c}{\partial t}\left(n_{r}, n_{s}, t\right) & =J\left(n_{r}^{-}, n_{s}, t\right)+J^{\prime}\left(n_{r}, n_{s}^{-}, t\right) .
\end{aligned}
$$

The densities of the model are defined as

$$
\begin{aligned}
& \int_{0}^{n_{s}} \int_{0}^{n_{r}} r c(r, s) d r d s=\rho_{\mathrm{I}}, \\
& \int_{0}^{n_{s}} \int_{0}^{n_{r}} s c(r, s) d r d s=\rho_{\mathrm{II}} .
\end{aligned}
$$

Thus, we have applied the density conservations (does not depend on time) instead of the monomer concentrations, and so the model is approximated by a differential algebraic equation [3].

The initial conditions of the system are

$$
\begin{aligned}
c(r, s, 0) & =0, \quad r+s>0, \\
c\left(0^{+}, 0,0\right) & =\rho_{\mathrm{I}}, \\
c\left(0,0^{+}, 0\right) & =\rho_{\mathrm{II}} .
\end{aligned}
$$

In the discrete form of the system, we assume

$$
\begin{aligned}
& c\left(r^{ \pm}, s, t\right) \approx c(r \pm \Delta r, s, t), \\
& c\left(r, s^{ \pm}, t\right) \approx c(r, s \pm \Delta s, t) .
\end{aligned}
$$

Hence, the discrete model is initialized with monomers, that is,

$$
\begin{aligned}
c(\Delta r, 0,0) & =\frac{\rho_{\mathrm{I}}}{\Delta r}, \\
c(0, \Delta s, 0) & =\frac{\rho_{\mathrm{II}}}{\Delta s}, \\
c(r, s, 0) & =0, \quad r \neq \Delta r \text { or } s \neq \Delta s .
\end{aligned}
$$


4. Numerical approximation. The truncated model is discretized by central difference in spaces and backward difference in time, that is, for

$$
\frac{\partial c}{\partial t}=-\frac{\partial J}{\partial r}-\frac{\partial J^{\prime}}{\partial s}, \quad(r, s) \in \mathbf{R}
$$

the discrete form at the mesh point $\left(r_{i}, s_{j}\right) \in \mathbf{R}$ and $t_{k}=k \Delta t\left(c_{i, j}^{k} \approx c\left(r_{i}, s_{j}, t_{k}\right)\right)$ is

$$
\frac{c_{i, j}^{k+1}-c_{i, j}^{k}}{\Delta t}=-\left(\frac{\partial J}{\partial r}\right)_{i, j}^{k+1}-\left(\frac{\partial J^{\prime}}{\partial s}\right)_{i, j}^{k+1},
$$

where, for example, the first term on the right-hand side can be formulated as follows:

$$
\begin{aligned}
& J(r, s, t)=-a(r, s) Q(r, s) c(\Delta r, 0, t)^{r+1} \frac{\partial}{\partial r}\left(\frac{c(r, s, t)}{Q(r, s) c(\Delta r, 0, t)^{r}}\right), \\
& \frac{\partial J}{\partial r}(r, s, t)=-\frac{\partial}{\partial r}\left(a(r, s) Q(r, s) c(\Delta r, 0, t)^{r+1}\right) \frac{\partial}{\partial r}\left(\frac{c(r, s, t)}{Q(r, s) c(\Delta r, 0, t)^{r}}\right) \\
&-a(r, s) Q(r, s) c(\Delta r, 0, t)^{r+1} \frac{\partial^{2}}{\partial r^{2}}\left(\frac{c(r, s, t)}{Q(r, s) c(\Delta r, 0, t)^{r}}\right), \\
&\left(\frac{\partial J}{\partial r}\right)_{i, j}^{k+1}= \frac{-1}{2 \Delta r}\left(a_{i+1, j} Q_{i+1, j}\left(c_{1,0}^{k+1}\right)^{r_{i}+\Delta r+1}-a_{i-1, j} Q_{i-1, j}\left(c_{1,0}^{k+1}\right)^{r_{i}-\Delta r+1}\right) \\
& \times \frac{1}{2 \Delta r}\left(\frac{c_{i+1, j}^{k+1}}{Q_{i+1, j}\left(c_{1,0}^{k+1}\right)^{r_{i}+\Delta r}}-\frac{c_{i-1, j}^{k+1}}{Q_{i-1, j}\left(c_{1,0}^{k+1}\right)^{r_{i}-\Delta r}}\right) \\
&-a_{i, j} Q_{i, j}\left(c_{1,0}^{k+1}\right)^{r_{i}+1} \frac{1}{\Delta r^{2}}\left[\frac{c_{i+1, j}^{k+1}}{Q_{i+1, j}\left(c_{1,0}^{k+1}\right)^{r_{i}+\Delta r}}-2 \frac{c_{i, j}^{k+1}}{Q_{i, j}\left(c_{1,0}^{k+1}\right)^{r_{i}}}\right. \\
&\left.+\frac{c_{i-1, j}^{k+1}}{Q_{i-1, j}\left(c_{1,0}^{k+1}\right)^{r_{i}-\Delta r}}\right] ;
\end{aligned}
$$

$\left(\partial J^{\prime} / \partial s\right)_{i, j}^{k+1}$ can be similarly evaluated.

For the boundary conditions, the discrete form of rates $J_{i, j}^{k+1}$ or $J_{i, j}^{\prime k+1}$ computed by the forward difference formula and the density conservation equations can be discretized as follows:

$$
\sum_{j} \sum_{i} r_{i} c_{i, j}^{k+1}=\rho_{\mathrm{I}}, \quad \sum_{j} \sum_{i} s_{j} c_{i, j}^{k+1}=\rho_{\mathrm{II}} .
$$




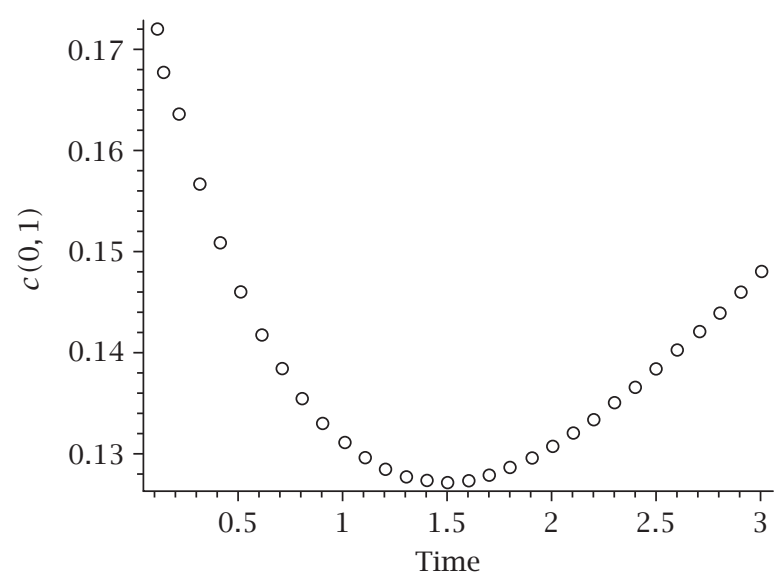

$\circ \circ \circ n_{r}=n_{s}=10$

(a)

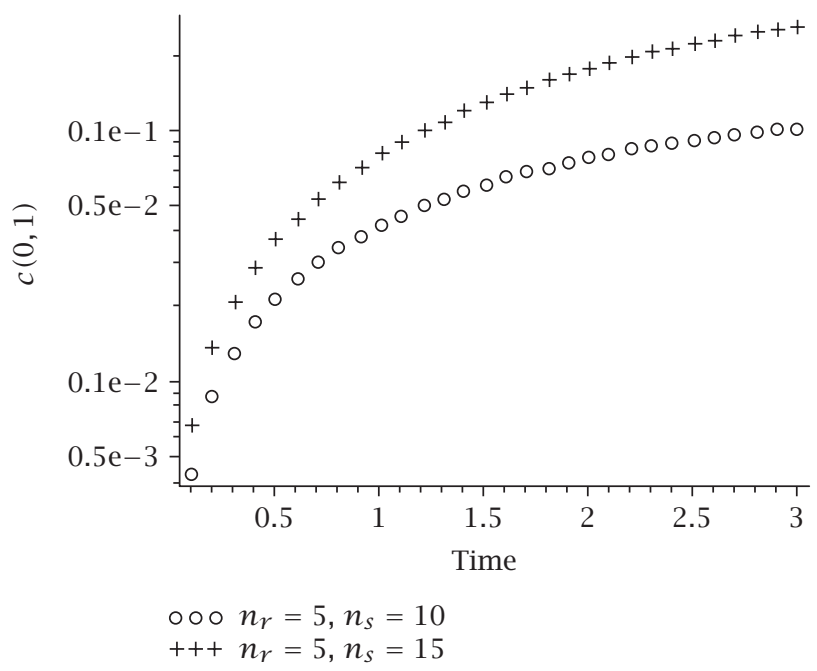

(b)

FIGURE 4.1. The monomer concentration of the two-component BeckerDöring system $c(0,1)$ against time. The densities are $\rho_{\mathrm{I}}(0)=\rho_{\mathrm{II}}(0)=1.5$ and kinetic coefficients are as in (3.14).

We write the two-dimensional array $c_{r, s}^{k+1}$ as a vector $y_{\ell}^{k+1}$ using the formula

$$
\ell=r\left(\left[\frac{n_{s}}{\Delta s}\right]+1\right)+s
$$

so the variables are ordered from $c_{0,1}^{k+1}=y_{1}^{k+1}$ to $c_{n_{r} / \Delta r, n_{s} / \Delta s}^{k+1}=y_{\left(n_{r} / \Delta r+1\right)\left(n_{s} / \Delta s+1\right)-1}^{k+1}$. 


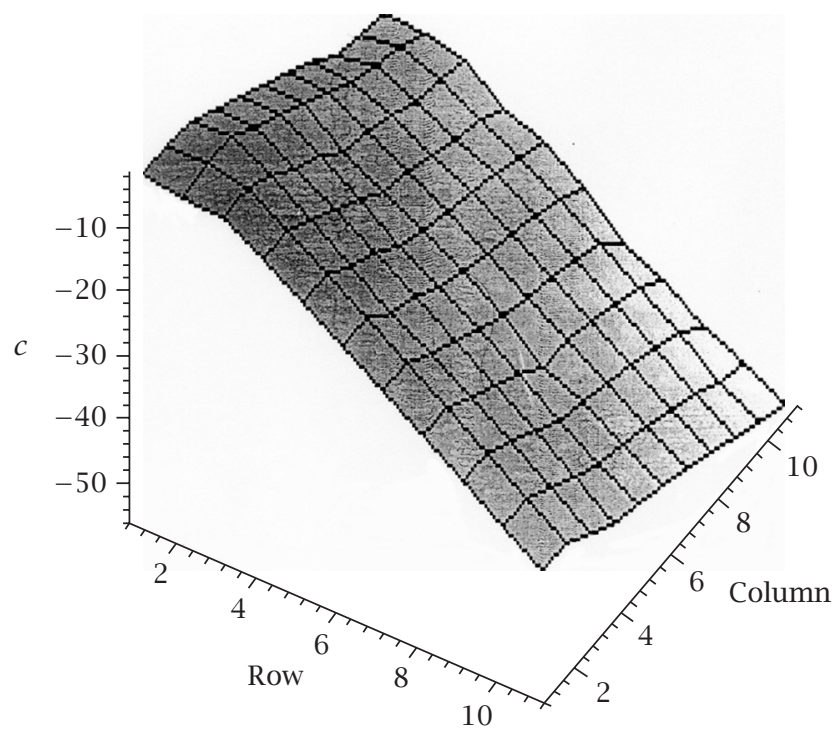

FIGURE 4.2. The cluster concentrations $c_{r, s}$ at time $t=3$ for $n_{r}=n_{s}=10$.

The discrete form of the model creates a nonlinear system of equations. We have solved them by Newton's method. The Jacobian matrix is very big, sparse, and has a regular structure. Because of the Becker-Döring system, the cluster size distribution changes when clusters gain or lose only monomers of each type. So the monomer concentrations appear in all equations from the definitions of $J_{r, s}$ and $J_{r, s}^{\prime}$. Therefore, the Jacobian matrix is a pentadiagonal matrix with nonzero elements in the first row and the first column (because of $y_{1}$ and (2.4)) and similarly in the $\left[n_{s} / \Delta s+1\right]$ th row and column.

Here we present the numerical approximation for square $\left(n_{r}=n_{s}\right)$ and rectangular $\left(n_{r} \neq n_{s}\right)$ models. They have different behaviors. In Figure 4.1, the monomer concentration of type-II particle $(c(0,1))$ is plotted against time. In Figure $4.1(\mathrm{a})$, the system size is $n_{r}=n_{s}=10$ and $\rho_{\mathrm{I}}=\rho_{\mathrm{II}}=1.5$, while in Figure $4.1(\mathrm{~b})$, the system is $5 \times 10$ and $5 \times 15$ with the same densities. The numerical approximation of the system at time $t=3$ is plotted against $r$ and $s$ in Figure 4.2. The PDE approximation needs more theoretical work. In our numerical experiment,

(1) when $\Delta r=\Delta s=1$, the results have a good accuracy;

(2) when $\Delta r<1, \Delta s<1$, the approximation does not work good and the roots of the nonlinear system are complex numbers after a few iterations. It should be related to the stiffness of the model;

(3) when $\Delta r>1, \Delta s>1$, the numerical results have a good stability and can be used for reduced models of a big truncated system.

For the reduced model, we guess that the nonuniform mesh with successive nodes around the monomers gives good results. The adaptive methods such as moving-mesh methods especially for the initial period of time give a useful nodes' distribution for time-dependent solutions. 


\section{REFERENCES}

[1] J. M. Ball, J. Carr, and O. Penrose, The Becker-Döring cluster equations: basic properties and asymptotic behaviour of solutions, Comm. Math. Phys. 104 (1986), no. 4, 657-692.

[2] R. Becker and W. Döring, Kinetische Behandlung der Keimbildung in übersättigten Dämpfern, Ann. Phys. 24 (1935), 719-752 (German).

[3] K. E. Brenan, S. L. Campbell, and L. R. Petzold, Numerical Solution of Initial-Value Problems in Differential-Algebraic Equations, Classics in Applied Mathematics, vol. 14, Society for Industrial and Applied Mathematics (SIAM), Pennsylvania, 1996.

[4] D. B. Duncan and A. R. Soheili, Approximating the Becker-Döring cluster equations, Appl. Numer. Math. 37 (2001), no. 1-2, 1-29.

[5] A. R. Soheili, Numerical analysis of the coagulation-fragmentation equations, Ph.D. thesis, Heriot-Watt University, Edinburgh, 1997.

Ali Reza Soheili: Department of Mathematics, Sistan \& Baluchestan University, Zahedan 98135, Iran

E-mail address: sohei1i@ahamoon.usb.ac.ir

Current address: Department of Mathematics, Simon Fraser University, Burnaby, BC, Canada V5A 1 S6

E-mail address: asohei1i@sfu.ca 


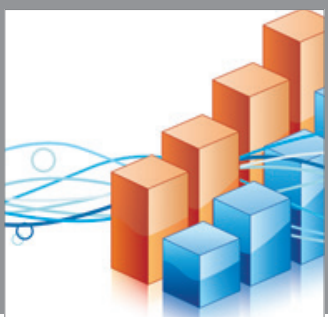

Advances in

Operations Research

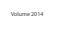

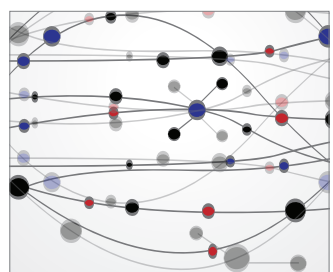

\section{The Scientific} World Journal
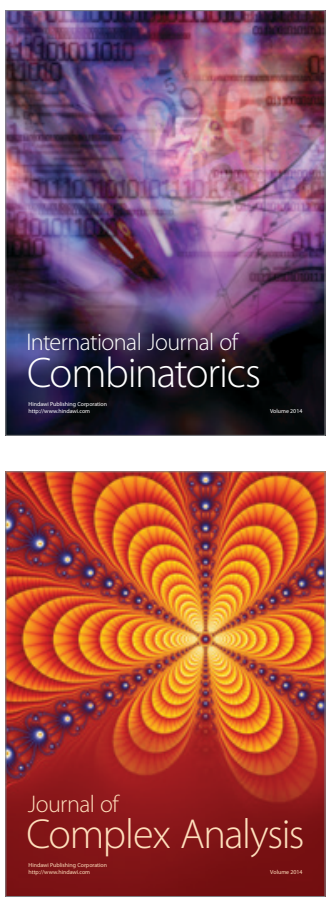

International Journal of

Mathematics and

Mathematical

Sciences
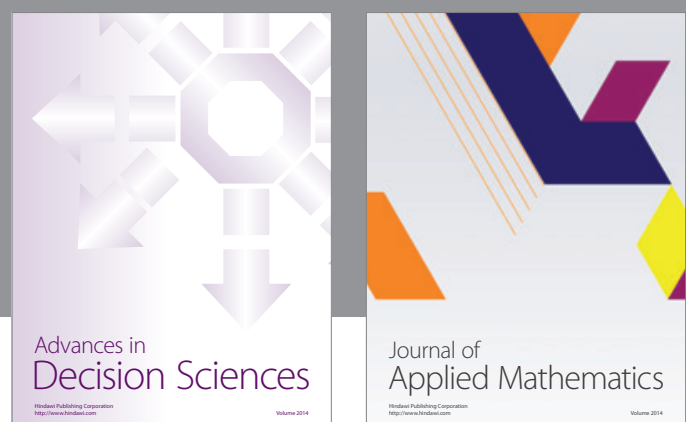

Journal of

Applied Mathematics
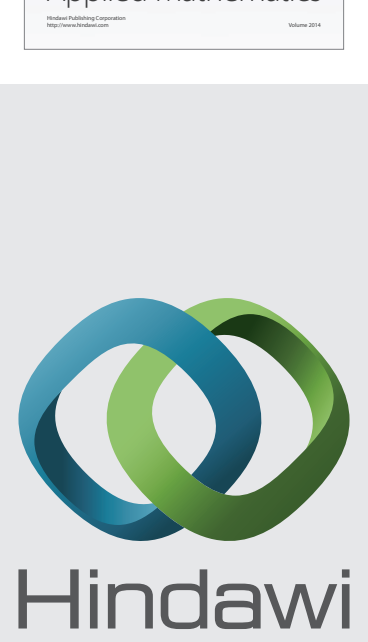

Submit your manuscripts at http://www.hindawi.com
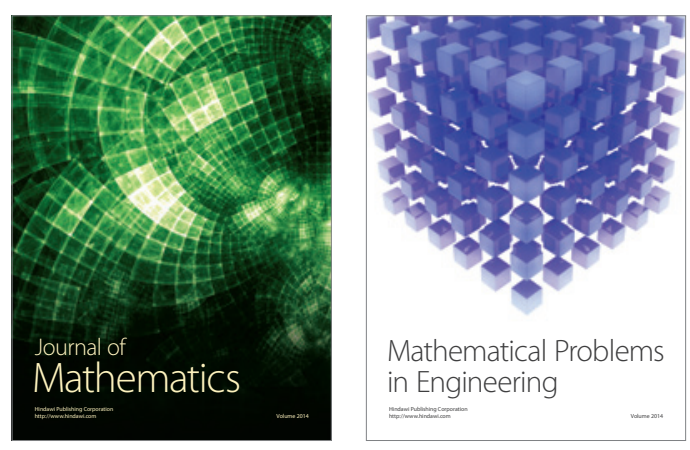

Mathematical Problems in Engineering
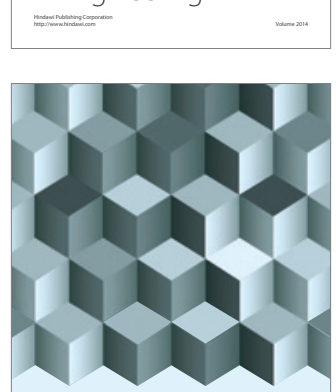

Journal of

Function Spaces
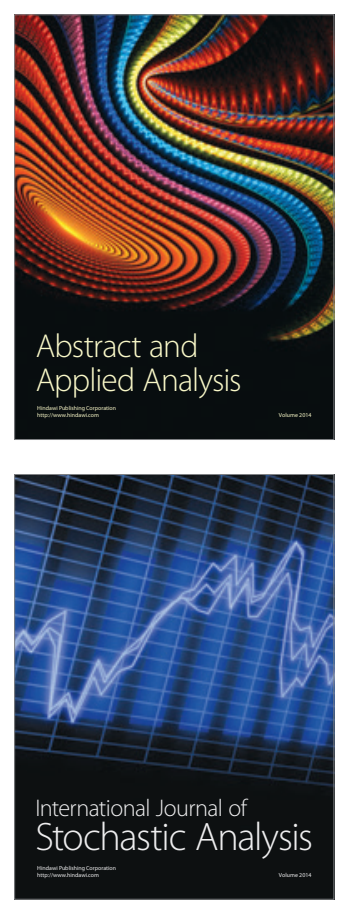

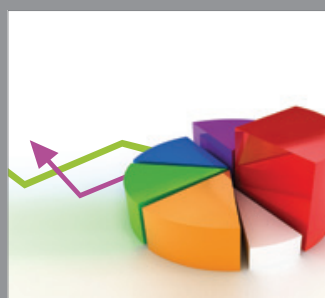

ournal of

Probability and Statistics

Promensencen
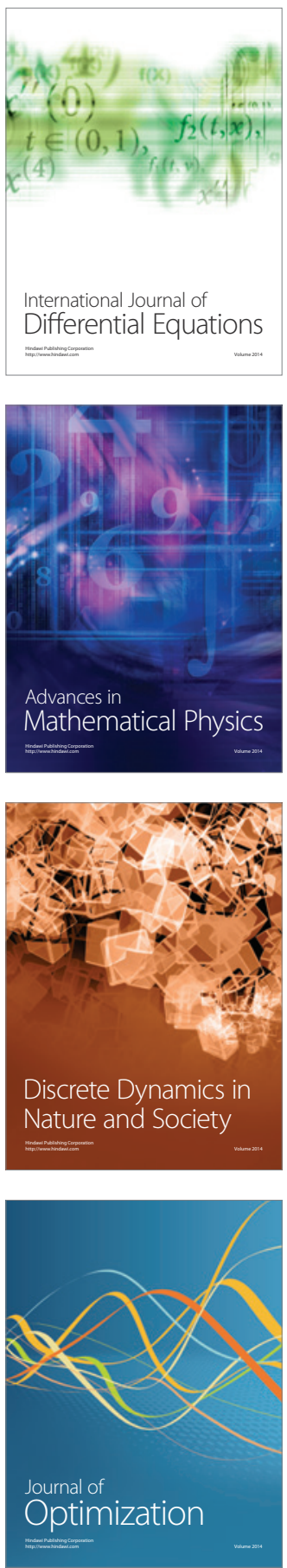